\title{
Search for energetic cosmic axions utilizing terrestrial/celestial magnetic fields
}

\author{
K. ZIOUTAS \\ Physics Department, University of Thessaloniki, GR-54006 Thessaloniki, Greece. \\ D. J. THOMPSON \\ NASA/Goddard Space Flight Center, Code 660, Greenbelt MD 207r1, USA.
}

AND

E. A. PASCHOS

Institut für Physik, Universität Dortmund, D-44221 Dortmund, Germany.

12. 8.1998

\begin{abstract}
Orbiting $\gamma$-detectors combined with the magnetic field of the Earth or the Sun can work parasitically as cosmic axion telescopes. The relatively short field lengths allow the axion-to-photon conversion to be coherent for $m_{\text {axion }} \sim 10^{-4} \mathrm{eV}$, if the axion kinetic energy is above $\sim 500 \mathrm{keV}$ (Earth's field), or, $\sim 50 \mathrm{MeV}$ (Sun's field), allowing thus to search for axions from $e^{+} e^{-}$annihilations, from supernova explosions, etc. With a detector angular resolution of $\sim 1^{\circ}$, a more efficient sky survey for energetic cosmic axions passing through the Sun can be performed. Axions or other axion-like particles might be created by the interaction of the cosmic radiation with the Sun, similarly to the axion searches in accelerator beam dump experiments; the enormous cosmic energy combined with the builtin coherent Primakoff effect might provide a sensitive detection scheme, being out of reach with accelerators. The axion signal will be an excess in $\gamma$-rays coming either from a specific celestial place behind the Sun, e.g. the Galactic Center, or, from any other direction in the sky being associated with a violent astrophysical event, e.g. a supernova. Earth bound detectors are also of potential interest. The axion scenario also applies to other stars or binary systems in the Universe, in particular to those with superstrong magnetic fields.
\end{abstract}

Emails : Konstantin.Zioutas@cern.ch

DJT@bartok.gsfc.nasa.gov

Paschos@hal1.physik.uni-dortmund.de 


\section{Introduction}

An attractive solution of the strong $\mathrm{CP}$ problem invokes a new symmetry, the PecceiQuinn (PQ) symmetry $\left(\mathrm{U}_{P Q}(1)\right)$. The spontaneous breaking of this new symmetry predicts the existence of a light neutral pseudoscalar particle, the axion, which is closely related to the neutral pion $[1,2]$. In fact, there are good reasons to believe that if the PQ mechanism is responsible for preserving $C P$ in the strong interactions, then the axion is the dark matter [3], i.e. axions may exist as primordial cosmic relics copiously produced in the early Universe, and eventually thermalized in a way similar to the $2.7^{\circ} \mathrm{K}$ cosmic background radiation. The axion also arises in supersymmetry and superstring theories. Thus, the axion is one of the leading and promising non-baryonic candidate for the ubiquitous dark matter in the universe [4]. Astrophysical observations and laboratory experiments leave open an axion rest mass window around $m_{a} \approx 10^{-4} \mathrm{eV}$ (within $\pm 1-2$ orders of magnitude). For all these reasons, axions have received much attention in elementary particle (astro)physics.

The axion decay into two photons $(a \rightarrow \gamma \gamma)$ is the reaction mainly used to search for them. Inside matter or in a magnetic field in vacuum, the axion couples to a virtual photon (Primakoff effect), producing a real photon $(\gamma)$, which can be detected :

$$
a+\gamma_{\text {virtual }} \rightleftharpoons \gamma
$$

The axion behaves like a very weakly interacting photon or pion $\left(\pi^{0}\right)$, and, in a reaction, it can replace a magnetic dipole $\gamma$ or a $\pi^{0}$. Energetic axions with mean thermal energy equal to $\sim 4 \mathrm{keV}[5,6]$ or $\sim 160 \mathrm{MeV}[7]$ are possibly copiously produced via the Primakoff effect inside the Sun or during a Supernova explosion, respectively. They also could be produced in astrophysical beam dumps [8], similar to the beam dump in accelerators, replacing energetic $\pi^{0}$ 's or $\gamma^{\prime}$ s in the electromagnetic/hadronic cascade reactions involved. Therefore, energetic axions have been searched already in high energy neutrino experiments [9]. A not so unusual beaming effect can compensate for the large distance to the Earth. In other words, it is not so unreasonable to expect high energy cosmic axions, beyond those expected to be emitted from a supernova. Finally, the existence of particles beyond the Standard Model with similar couplings can not be excluded.

\section{Previous work}

The stimulation for this proposal comes from two recent works, which appeared almost simultaneously by two groups [7] searching for energetic axions from SN1987A with data from orbiting detectors. However, they could have provided an axion signature for specific parameter values, i.e., for $m_{a} \leq 10^{-9} \mathrm{eV}$ and an axion-to-photon coupling constant $g_{a \gamma \gamma} \geq 3 \cdot 10^{-12} \mathrm{GeV}^{-1}$ assuming $\sim 1 \mathrm{kpc}=3 \cdot 10^{19} \mathrm{~m}$ galactic magnetic field length of $\sim 2 \mu$ gauss, where the axion-to-photon conversion via the coherent Primakoff effect can take place. In such a case, an orbiting detector pointing to the SN1987A position in the sky should have measured an excess of energetic photons during this historical supernova observation on Earth, provided the number of the emitted axions were sufficient to trigger the detector. These two groups [7] have also estimated the hypothetical thermal axion spectrum from the supernova, with the main unknown being the axion-to-photon coupling constant $\left(g_{a \gamma \gamma}\right)$. 


\section{The suggestion}

The concept of this work is similar with that given in ref. [7]. The main difference is the choise of the magnetic field between the axion source and the $\gamma$-ray detector, where the axion conversion takes place; we suggest to use the solar $(\sim 2$ gauss $)$ and/or the terrestrial $(\sim 0.5$ gauss $)$ magnetic fields. In order to justify this choise, we give below two relations, which describe the axion interaction inside a static magnetic field.

Firstly, the transverse magnetic field strength $B$, its length $L$ and the axion-to-photon coupling constant $g_{a \gamma \gamma}$ are the fundamental parameters in the calculation of the coherent axion-to-photon conversion inside $\mathrm{B}$. The probability to have a photon from an energetic axion entering perpendicularly a $1 \mathrm{~T} \cdot \mathrm{m}$ magnetic field , is $[5,6,10]$

$$
P_{a \rightarrow \gamma}=\left(\frac{g B L}{2}\right)^{2}=2.5 \times 10^{-21}\left[\frac{\mathrm{B}}{1 T} \frac{\mathrm{L}}{1 m} \frac{\mathrm{g}_{\mathrm{a} \gamma \gamma}}{10^{-10} \mathrm{GeV}^{-1}}\right]^{2},
$$

It is interesting to notice the $(B \cdot L)^{2}$ dependence of the coherent axion-to-photon conversion rate.

Secondly, for massive axions, in order to fullfil the coherence relation (2), i.e. to exclude deconstructive axion-photon interference over the magnetic field length $(\mathrm{L})$, the axion rest mass $\left(m_{a}\right)$ and its total energy $\left(E_{a}=E_{\gamma}=\hbar \omega\right)$ must satisfy a second relation $[5,6]$

$$
L \leq \frac{(2 \pi \hbar c) \cdot(\hbar \omega)}{\left|m_{a}^{2}-m_{\gamma}^{2}\right| c^{4}}
$$

In this relation, $m_{\gamma}$ reflects the acquired mass of the photons inside matter, which depends on the electron density : $m_{\gamma}[\mu e V] \approx 0.37 \times \sqrt{\rho_{e}\left[10^{8} / \mathrm{cm}^{3}\right]}$. Thus, for an electron density $\rho_{e} \leq 10^{8} / \mathrm{cm}^{3}$ (i.e. $m_{\gamma} \ll m_{a}$ ), which can be the case with the considered terrestrial and solar regions [11], relation (3) becomes

$$
L \leq \frac{(2 \pi \hbar c) \cdot(\hbar \omega)}{m_{a}^{2} c^{4}}
$$

Inserting $m_{a} \approx 10^{-4} \mathrm{eV}$ and $\bar{E}_{a} \approx 160 \mathrm{MeV}$ into this relation, the resulting coherence length (for $E_{a} \geq 50 \mathrm{MeV}$ ) can be as large as

$$
L \approx 4 \cdot 10^{9} m \approx 6 R_{\odot}
$$

Similarly, for $m_{a} \approx 10^{-4} \mathrm{eV}$ and $E_{a}=511 \mathrm{keV}$ it follows :

$$
L \approx 10^{7} m \approx 1 R_{\oplus}
$$

One should notice that the study in ref. [7] is sensitive to axions with rest mass below $10^{-9} \mathrm{eV}$, because they used the much longer coherent-galactic-magnetic field $(\sim 1 \mathrm{kpc})$; this mass range is far below the open axion mass window $\left(m_{a} \approx 10^{-(4 \pm 2)} \mathrm{eV}\right)$.

Thus, taken into account the coherence lengths given in relations (5) and (6), the Earth's magnetic field is in this respect just appropriate for an axion threshold energy of $\sim 500 \mathrm{keV}$, while the more efficient solar magnetic field, fits to high energy axions $\left(E_{a} \geq 50 \mathrm{MeV}\right)$. We take for the terrestrial and solar $B \cdot L$ values

$$
(B \cdot L)_{\oplus} \approx 300 T \cdot m, \quad \text { and }, \quad(B \cdot L)_{\odot} \approx 3 \cdot 10^{4} T \cdot m
$$


respectively. It is worth mentioning that solar flares with $\sim$ kgauss magnetic fields and some $10^{3} \mathrm{~km}$ in size can have $B \cdot L \approx 10^{5}-10^{6} \mathrm{~T} \cdot \mathrm{m}$. For comparison, one should keep in mind that laboratory magnetic axion spectrometers use magnetic fields with $B \cdot L \approx 10-100 \mathrm{~T} \cdot \mathrm{m}$ at best $[5,6,12]$; inspite of the $\sim 2-10 \mathrm{~T}$ field strength, they have to be anyhow short (s. relation (4)), because of the much lower expected solar axion energy $(\sim 4 \mathrm{keV})$.

With relation (2) we can estimate the conversion probability $P_{a \rightarrow \gamma}$ for an energetic axion propagating inside the Sun's, or, Earth's magnetic field :

$$
P_{a \rightarrow \gamma}^{\odot}=2.5 \times 10^{-12} \cdot\left[\frac{(\mathrm{B} \cdot \mathrm{L})}{\left(3 \cdot 10^{4} \mathrm{~T} \cdot \mathrm{m}\right)} \frac{\mathrm{g}_{\mathrm{a} \gamma \gamma}}{10^{-10} \mathrm{GeV}^{-1}}\right]^{2}
$$

and

$$
P_{a \rightarrow \gamma}^{\oplus}=2.5 \times 10^{-16} \cdot\left[\frac{(\mathrm{B} \cdot \mathrm{L})}{(300 \mathrm{~T} \cdot \mathrm{m})} \frac{\mathrm{g}_{\mathrm{a} \gamma \gamma}}{10^{-10} \mathrm{GeV}^{-1}}\right]^{2}
$$

The Earth's magnetic field allows in principle for a simultaneous $\sim 2 \pi$ survey of the sky, even though the field of view (f.o.v.) of an individual (orbiting) detector is smaller. This is not the case with the solar field; as the Earth and the Sun change continuously their orientation in space, one can scan 'through the sun' a big part of the sky with $\sim 0.5^{\circ}$ opening angle. We consider an effective solar axion conversion region of a few solar radii (see relation (5)) including the Sun.

To be more specific, we give a numerical example for a supernova, which might be taken as reference for other violent astrophysical events. The expected integrated axion flux $\left(\Phi_{a}\right)$ on Earth from a supernova explosion, which lasts some $\sim 10-20$ seconds and is at a distance $D \approx 6 k p c$, is [7]

$$
\Phi_{a}\left(\bar{E}_{a} \approx 160 \mathrm{MeV}\right) \approx 2.5 \cdot 10^{9} \text { axions } \cdot \mathrm{cm}^{-2} \cdot\left[\frac{g_{a \gamma \gamma}}{10^{-10} \mathrm{GeV}^{-1}}\right]^{2} \cdot\left[\frac{6 \mathrm{kpc}}{\mathrm{D}}\right]^{2},
$$

with the axions created from the scattering of thermal photons on protons through the Primakoff effect $(p \gamma \rightarrow p a)$. The energy released by the axions is $\sim 100$ times smaller than the energy released through the escaping neutrino burst.

Combining relations (8), (9) and (10), we estimate the signal $S^{\odot}=P_{a \rightarrow \gamma}^{\odot} \times \Phi_{a}$ for an orbiting detector. The flux of axions from the supernova converts into photons of $\sim 160$ $\mathrm{MeV}$ in the solar field and gives the final signal :

$$
S^{\odot} \approx 6 \cdot 10^{-3} \mathrm{~cm}^{-2} \cdot\left[\frac{g_{a \gamma \gamma}}{10^{-10} \mathrm{GeV}^{-1}}\right]^{4} \approx 8 \mathrm{~cm}^{-2} \cdot\left[\frac{g_{a \gamma \gamma}}{6 \cdot 10^{-10} \mathrm{GeV}^{-1}}\right]^{4}
$$

Similarly, the intervening terrestrial field yields :

$$
S^{\oplus} \approx 6 \cdot 10^{-7} \mathrm{~cm}^{-2} \cdot\left[\frac{g_{a \gamma \gamma}}{10^{-10} \mathrm{GeV}-1}\right]^{4} \approx 8 \cdot 10^{-4} \mathrm{~cm}^{-2} \cdot\left[\frac{g_{a \gamma \gamma}}{6 \cdot 10^{-10} \mathrm{GeV}^{-1}}\right]^{4}
$$

Notice the $\left(g_{a \gamma \gamma}\right)^{4}$ dependence of the signal, while $g_{a \gamma \gamma} \leq 6 \cdot 10^{-10} \mathrm{GeV}^{-1}$ is the presently best experimental limit for the coupling constant [12].

In a supernova explosion electrons and positrons are created by the interacting neutrinos above the neutrinosphere $\left(\rho \leq 10^{11} \mathrm{~g} / \mathrm{cm}^{3}\right)$ via the reactions $\nu \bar{\nu} \rightarrow e^{+} e^{-}, \nu_{e} p \rightarrow p e^{-}$ and $\overline{\nu_{e}} p \rightarrow n e^{+}$, the dominant processes which generate neutrino opacity [13]. A similar situation might arise in the case of two merging neutron stars [14]. While the annihilation 
$\gamma$-line of those positrons is completely shielded, axions created in the $e^{+} e^{-}$-annihilation can escape. It is worth remembering that $511 \mathrm{keV}$ axion search, following the reaction $e^{+} e^{-} \rightarrow \gamma a$, has been performed already in laboratory experiments with positron sources [22]. Needless to say that the same process could occur with those obscured intense positron places in the Universe, while the intervening terrestrial or any other magnetic field works as axion-to-photon converter (s. section 5.).

Obviously, the solar signature $\left(S^{\odot}\right)$ will show-up when the detector, the Sun and the axion source are aligned within $\sim 0.5^{\circ}$, in which case the f.o.v. of the detector sees the axion source. The geometry with the Earth's magnetic field is actually free from such constraints, however, the conversion efficiency is smaller (s. relation (8) and (9)). In other words, taking into account the suggested axion conversion inside the terrestrial or solar magnetic field in the evaluation of $\gamma$-ray data, an orbiting gamma detector is also an axion telescope, scaning continuously the sky for such events.

Background: The isotropic cosmic $\gamma$-ray flux above $\sim 100 \mathrm{MeV}$ is $\sim 10^{-5} \gamma^{\prime} \mathrm{s} / \mathrm{cm}^{2} \cdot \mathrm{s} \cdot \mathrm{sr}$ while that from the Galactic plane is by a factor of $\sim 10$ higher [15]. Furthermore, the electromagnetic/hadronic interaction of the cosmic radiation with the Sun must give rise to energetic photons, and in addition there are $\gamma$-rays from solar activity, e.g. flares [16]. Sofar, the orbiting EGRET detector measured an excess of high-energy gamma radiation from the moon. The lunar flux above $100 \mathrm{MeV}$ is $\sim 5 \cdot 10^{-7} \gamma^{\prime} \mathrm{s} / \mathrm{cm}^{2} \cdot \mathrm{s}$, while the limit obtained for the quiet Sun is below $\sim 2 \cdot 10^{-7} \gamma^{\prime} s / \mathrm{cm}^{2} \cdot s[15]$. The $511 \mathrm{keV}$ flux from the Galactic Center is $\sim 2 \cdot 10^{-4} \gamma^{\prime} \mathrm{s} / \mathrm{cm}^{2} \cdot \mathrm{s}$, with the $3 \gamma$ annihilation continuum below 511 $\mathrm{keV}$ being by a factor $\sim 5$ higher [17]. The observed Galactic positron annihilation rate is $\geq 10^{43} / \mathrm{sec}$ [18]; those positrons can be created through the decay from radioactive nuclei produced by supernovae, novae, and the massive Wolf-Rayet stars with violent surface activity, but also from $\gamma-\gamma$ pair production in the vicinity of an accreting black hole [17], whose violence is probably without analog.

The axion signature : a) The axion signal associated with the Earth's field will be burst-like and in coincidence with some violent astrophysical event, e.g. a supernova. This is a type of signal discussed in ref. [7]. b) Cosmic axions converted in the solar field can be identified as an excess of $\gamma$-rays coming from the region of the Sun, provided the direction from the detector to the Sun points at the same time to a specific source outside the Sun, e.g. the Galactic Center. If a $\gamma$-ray excess is seen coincident with a radio/optical/x-ray flare, then the gamma radiation could be from the flare. These flares are monitored continuously by the GOES satellite, so screening out solar flare events is straightforward. ${ }^{1}$

\section{Orbiting and Earth bound detectors}

The requirements for the high energy gamma detector are actually obvious from the previous considerations of the potential cosmic axion signature. We give here in summary the main optimum specifications : 1) threshold energy $\sim 0.5 / 50 \mathrm{MeV}$, with a modest energy resolution being sufficient, 2) background suppression, 3) large detector aperture/f.o.v., 4) angular resolution of a few degrees and 5) photon identification.

For example, EGRET satisfies most of the above requirements. The planned GLAST project [20], with an effective area $\sim 8000 \mathrm{~cm}^{2}$ (above $1 \mathrm{GeV}$ ), f.o.v. covering $\sim 20 \%$ of the sky, angular resolution of $2.5^{\circ}$ (at $100 \mathrm{MeV}$ ) and $0.1^{\circ}$ (above $10 \mathrm{GeV}$ ), energy range

\footnotetext{
${ }^{1}$ The rather strong magnetic fields in solar flares are also quite suitable axion converters. With better understanding of solar flares the question arises whether $\gamma$-rays from axions could be identified within solar flares observations (s. ref. [19] for very low-mass axions in the x-ray region).
} 
$10 \mathrm{MeV}$ to $300 \mathrm{GeV}$, energy resolution $\sim 10 \%$, will be a factor of $\sim 30$ more sensitive than EGRET and it can become the best potential high energy cosmic axion antenna in orbit.

For the search of the $511 \mathrm{keV}$ line different orbiting detectors come into question. The OSSE detector is certainly the best instrument in orbit since 1991 [21]; it has $\sim 2000 \mathrm{~cm}^{2}$ aperture at $511 \mathrm{keV}$, f.o.v. $3.8^{\circ} \times 11.4^{\circ}$, an energy resolution of $8 \%$ at 661 $\mathrm{keV}$, while its energy range from $50 \mathrm{keV}$ to $10 \mathrm{MeV}$ is just complementary to the GLAST performance. The planned European mission INTEGRAL will also be sensitive to 511 $\mathrm{keV}$ axions (energy range $15 \mathrm{keV}$ to $10 \mathrm{MeV}$ ); its f.o.v. will be $4.8^{\circ}-16^{\circ}$ and its targets of observation will include the Galactic Center. Further, one should reconsider data from detectors, which have had within their f.o.v. the region of SN1987A, eventhough there is as yet no axion flux estimate at $511 \mathrm{keV}$ from astrophysical places like a supernova or other source in the sky.

Following the same reasoning at high energies, Earth bound detectors come also into question, provided they have the required photon identification signature, and, the directional reconstruction of the incident photon. However, a sky survey 'through the Sun' requires a solar blind $\gamma$-detector, i.e., the detection technique can not use atmospheric Cherenkov or scintillation light in the visible. The high energy $\gamma$-radiation seen from the Moon with EGRET [15] and the observed shadowing of cosmic rays by the Sun and the Moon, with surface [23] and deep underground detectors [24], show the feasibility of this kind of investigations. ${ }^{2}$

\section{Discussion}

We have used a supernova explosion as a representative astrophysical violent event, for which a possible axion involvement below $\sim 300 \mathrm{MeV}$ has been estimated already quantitatively. However, it is reasonable to assume that if axions or any other axion-like particles exist, then, they could be copiously created in other flare stars or in transient brightenings, which we know to release comparable or greater energy. This work suggests primarily to utilize the terrestrial and the solar magnetic field as axion-to-photon converters, in order to perform with orbiting detectors a sky survey, searching for cosmic axions with energy above $\sim 0.5 / 50 \mathrm{MeV}$.

An axion signature can show-up either as a burst, or as an event rate being proportional to the intervening $(B \cdot L)^{2}$ value between the hypothetical axion source and the detector. This can be the case, for example, with the Earth's field by comparing gamma rates observed at different distances from the Earth. For example, the INTEGRAL mission will fly between $\sim 10000 \mathrm{~km}$ and $\sim 150000 \mathrm{~km}$.

\footnotetext{
${ }^{2}$ Similarly, in accelerators, high energy detector systems with their inner charged particle tracking magnetic field surrounded by $\sim 4 \pi$ electromagnetic calorimeter can also perform this kind of investigations. In fact, they can operate parasitically for this purpose, provided they have a built-in trigger, which allows to register any cosmic ray hitting the detector when the accelerator is OFF, or between beam crossings. The potential axion signature, i.e. isolated energetic photons coming from the magnetic field region, will be of interest independent on the time of occurence or direction of arrival. Fortunately, background measurements can be performed by switching OFF the magnetic field. The effective $B \cdot L$ value is usually $\approx 1-10 \mathrm{~T} \cdot \mathrm{m}$, while the $\sim 1 \mathrm{~m}$ transverse field length makes them coherent high energy axion converter for an axion rest mass up to 1-10 eV; their geometry allows to perform, however, simultaneously a full sky high-energy axion survey. To the best of our knowledge, none magnetic detector was ON to convert energetic or $\sim 511 \mathrm{keV}$ axions during SN1987A.

A search for energetic axions can also be performed with the powerful accelerator bending magnets [6], which have $B \cdot L \approx 100 T \cdot m \approx(B \cdot L)_{\oplus}$ and a built-in angular resolution/acceptance of $\leq 0.5^{\circ}$.
} 
We also mention a few other places in the sky as potential sources for axions. a) astrophysical 'beam dumps' [8], e.g. relativistic 'fireballs', jets, etc., which seem to be associated with the as yet enigmatic Gamma Ray Bursts (GRBs); the most powerful explosions in the Universe after the Big Bang : the released energy (some $10^{52 \pm 2}$ ergs) is probably much more than that from a supernova explosion [25, 26].

b) the Galactic Center (GC), which is one of the most dynamical regions in our Galaxy, with numerous activities remaining hidden. For example, EGRET observed a $\gamma$-ray source luminosity $L \approx 10^{4} L_{\odot}$ in the energy range $30 \mathrm{MeV}$ to $20 \mathrm{GeV}$ [28]. Further, the recent OSSE discovery of a giant cloud of positrons extending $\sim 1 k p c$ above the GC was unexpected, since antimatter is thought to be relatively rare in the Universe.

c) close binaries, e.g. cataclysmic variables, hypernova [25], etc.

Inspite of missing predictive theoretical models for energetic cosmic axions beyond those to be emitted from a supernova, we propose to implement this kind of investigations in the different photon detectors in orbit or on Earth. The realization of these investigations require only an appropriate data re-evaluation and/or trigger. Such sky surveys might unravel novel physical processes occuring deep inside a star or our Galaxy.

For cosmic axions with energy far above that expected to be emitted from a supernova, i.e. $E_{a} \gg 10^{8} \mathrm{eV}$, also the $\sim k p c$ galactic magnetic fields considered in ref. [7] can be very efficient axion converters for an axion rest mass in the open axion mass range (s. rel. (4)). Of course, no orbiting detector can measure the energy of such very energetic photons. However, for the purpose of this suggestion, a mere photon identification might well be sufficient as a first signature.

An additional perspective, of no minor importance, is the possibility to create and detect, during the quasi 'beam dump' of the cosmic radiation into the Sun, axions or other new weakly interacting particles with similar couplings [27]. Because of the huge thickness of the Sun, even a very weakly interacting component of the cosmic radiation might interact there, which is beyond reach in accelerator beam dump experiments. In addition, the advantage of this configuration compared with accelerator experiments is the much higher cosmic energy, combined with the built-in highly efficient coherent axion-to-photon conversion inside the solar magnetic field.

Finally, inspired by the celebrated microlensing phenomenon [29], it does not escape our attention that the considered alignement between the cosmic axion-source, the solar field and the $\gamma$-detector can also happen with another magnetic star in the sky replacing the Sun [30]. The axion interaction can be enhanced in stars having strong magnetic fields, e.g. for $B \geq 10^{12}$ gauss $[25,31]$ around a neutron star, or $B \leq 10^{9}$ gauss around a white dwarf [30], where the $B \cdot L$-values can be above $\sim 10^{12} \mathrm{~T} \cdot \mathrm{m}$; for certain parameter values, the conversion efficiency axion-to-photon, and vice versa, might reach reasonable values. In particular, the axion scenario could be present in eclipsing (close) binaries with superstrong magnetic fields, whose configuration might imply a high degree of alignment with the Earth. The small size of a neutron star allows coherence Primakoff interaction also in the x-ray region [32]. Therefore, if axions exist, they could be responsible for some of the time variable or transient cosmic $\gamma$-ray sources, including GRBs and Soft Gamma-ray Repeaters.

\section{Acknowledgements}

Two of us (E.A.P. and K.Z.) like to thank NATO for a cooperative research grant. 


\section{References}

[1] R.D. Peccei, H.R. Quinn, Phys. Rev. D16 (1977); Phys. Rev. Lett. 38 (1977) 1440.

[2] F. Wilczek, Phys. Rev. Lett. 40 (1978) 279; S. Weinberg, ibid 48 (1978) 223.

[3] M. Kamionkowski, CU-TP-866, CAL-648, hep-ph/9710467 (24. 10. 1997).

[4] G. G. Raffelt, Proc. XVIII Intern. Conference on Neutrino Physics and Astrophysics, ed. Y. Suzuki and Y. Totsuka, Takayama, Japan (June 1998). See also hep-ph/9806506 (27.6.1998), and references therein.

[5] D. M. Lazarus, G. C. Smith, R. Cameron, A. C. Melissinos, G. Ruoso, Y. K. Semertzidis, F. A. Nezrick, Phys. Rev. Lett. 69 (1992) 2333.

[6] K. Zioutas et al., astro-ph/9801176 (18. 1. 1998), N.I.M. A (1998) in press.

[7] E. Masso and R. Toldra, Phys. Rev. D52 (1995) 1755, J. A. Grifols, E. Masso, R. Toldra, Phys. Rev. Lett. 77 (1996) 2372, E. Masso, astro-ph/9704056 and J. W. Brockway, E. D. Carlson, G. G. Raffelt, Phys. Lett. B383 (1996) 439.

[8] T. K. Gaisser, Cosmic Rays and Particle Physics, Cambridge University Press (1990) 177.

[9] T. Hansl et al., Phys. Lett. 74B (1978) 139, P. Alibran et al., ibid 74B (1978) 134, P.C. Bosetti et al., ibid 74B (1978) 143, F. Bergsma et al., ibid 157B (1985) 458.

[10] G. Raffelt and L. Stodolsky, Phys. Rev. D37 (1988) 1237;

G. Raffelt, Phys. Rev. D33 (1986) 897.

[11] C. W. Allen, Astrophysical Quantities, 2nd edition, University of London, The Athlone Press (1963) pp. 133, 176.

[12] S. Moriyama, M. Minowa, T. Namba, Y. Inone, Y. Takusu, A. Yamamoto, RESCEU-23/98, hep-ex/9805026, submitt. to Phys. Lett. B (1998).

[13] R. C. Duncan, S. L. Shapiro, I. Wasserman, ApJ. 309 (1986) 141;

S. E. Woosley, E. Baron, ApJ. 391 (1992) 228;

S. E. Woosley, Astron. \& Astrophys. Suppl. Series 97 (1993) 205.

[14] D. Eichler et al., Nature 340 (1989) 126.

[15] P. S. Sreekumar et al., ApJ. 494 (1998) 523; D. J. Thompson, D. L. Bertsch, D. J. Morris, R. Mukherjee, J. Geophys. Res. - Space Phys. 102, A7 (1997) 14735; S. D. Hunter et al., ApJ. 481 (1997) 205.

[16] E.g. : G. Kanbach et al., Astron. \& Astrophys. Suppl. Series 97 (1993) 349;

N. G. Leikov et al., ibid 97 (1993) 345.

[17] W. R. Purcell et al., ApJ. Lett. 417 (1993) 738, ApJ. 491 (1997) 725;

R. L. Kinzer et al., Astron. \& Astrophys. 120 (1996) C317. 
[18] R. Ramaty, R. E. Lingenfelter, Astron. \& Astrophys. Suppl. Series 97 (1993) 127.

[19] E. D. Carlson and L.-S. Tseng, Report HUTP-95/A025 and hep-ph/9507345.

[20] P. F. Michelson, SPIE Proc. 2806 (1996) 31.

[21] W. N. Johnson et al., ApJ. Suppl. 86 (1993) 693.

[22] E.g.: U. Amaldi et al., Phys. Lett. 153B (1985) 444; S. Orito et al., Phys. Rev. Lett. 63 (1989) 597; M. V. Akopyan et al., Phys. Lett. B272 (1991) 443; S. Asai et al., Phys. Rev. Lett. 66 (1991) 2440; T. Mitsui et al., Phys. Rev. Lett. 70 (1993) 2265; T. Maeno et al., Phys. Lett. B351 (1995) 574; M. Skalsey, R. S. Conti Phys. Rev. A55 (1997) 984.

[23] M. Ambrosio et al., ApJ. 464 (1996) 954, ibid 415 (1993) L147, Phys. Rev. D47 (1993) 2675; A. Borione et al., Phys. Rev. D49 (1994) 1171.

[24] M. Ambrosio et al., The MACRO collaboration, hep-ex/9807006 (7. 7. 1998).

[25] B. Paczyński, astro-ph/9706232 (23. 6. 1997), and, 4th Huntsville GRB Symposium/Huntsville, Sept. 1997, s. also astro-ph/9712123 (8. 12. 1997).

[26] A. Dar, ApJ. 500 (1998) L93.

[27] S. N. Gninenko and N. V. Krasnikov, Phys. Lett. B427 (1998) 307;

J. Altegoer et al., The NOMAD collaboration, Phys. Lett. B428 (1998) 197.

[28] H. A. Mayer-Hasselwander et al., Astron. \& Astrophys. 335 (1998) 161;

s. also D. H. Hartmann et al., astro-ph/9709029 (3.9.1997).

[29] B. Paczyński, ApJ. 304 (1986) 1.

[30] G. G. Raffelt, Stars as Laboratories for Fundamental Physics, The University of Chicago Press, Chicago \& London (1996) p.185.

[31] R. C. Duncan and C. Thompson, ApJ. 392 (1992) L9.

[32] D. E. Morris, Phys. Rev. D34 (1986) 843.

This work considers X-ray emission $\left(E_{\gamma} \approx 50 \mathrm{keV}\right)$ from the magnetosphere of a pulsar, if axions are thermally created in its core (s. also ref. [30]). 